\title{
Color stability of FCC gasoline
}

\author{
Song Lechun, Li Peibo, Lu Liang, Chen Jihai, Zhou Yulu, Xiang Yuzhi and \\ Xia Daohong*
}

State Key Laboratory of Heavy Oil Processing, China University of Petroleum, Qingdao, Shangdong 266580, China

(C) China University of Petroleum (Beijing) and Springer-Verlag Berlin Heidelberg 2012

\begin{abstract}
The color changes of one representative FCC gasoline were studied. The red substance in the FCC gasoline was concentrated and separated by chromatography and analyzed by elemental analysis and gas chromatography-mass spectrometry (GC-MS). The main components of the red substance were found to be aromatic amines. Complexes formed from quinones and aromatic amines are the reason why gasoline being red, and acids can destroy the complex by reaction with aromatic amines leading to decoloration of red gasoline. A mechanism for the color change of gasoline is proposed.
\end{abstract}

Key words: Gasoline, color stability, phenol, basic nitrogen compounds

\section{Introduction}

In recent years, the gasoline produced by China's refineries darkened and showed poor color stability, which seriously affected the quality of gasoline. While there are many reports on color stability of fluid catalytic cracking (FCC) diesel oil, investigation of FCC gasoline has been rarely reported. The stability of gasoline can be defined as the properties of maintaining its original quality under storage and use conditions. Generally, gasoline stability is related with physical, chemical and thermal factors in storage and processing.

In addition to olefins, sulfur and nitrogen compounds (Thompson et al, 1949), conjugated dienes seriously affect the stability of gasoline (Andziulis, 2006; Pereira and Pasa, 2006; Zanier, 1998). The formation of gum and the decrease of the induction period of gasoline during storage, which affects the stability of gasoline, has been widely studied (Wu et al, 2008a). However few reports are available on the color changes of FCC gasoline. It is reported that nitrogen compounds, especially basic nitrogen compounds could induce the color of diesel to darken (Batts and Fathoni, 1991; Thompson et al, 1951; Zuo, 2005). In most studies the color changes of diesel are attributed to chemical reactions, especially the oxidation of the basic nitrogen compounds under the catalysis of acidic materials in diesel during storage. As the mechanism for color change of FCC gasoline has not yet been reported, investigation of it is needed.

\section{Experimental section}

\subsection{Materials}

The red gasoline used in experiment is the finished FCC

*Corresponding author. email: xiadh@upc.edu.cn

Received May 18, 2011 gasoline from one refinery in China, which is fresh gasoline from the finished pot and contains additives.

\subsection{Separation and purification of the red substance in the FCC gasoline}

A chromatographic column $(0.40 \mathrm{~cm}$ in diameter and $15.00 \mathrm{~cm}$ in length) was filled with activated silica gel or neutral alumina $(0.882 \mathrm{~g})$ and $650 \mathrm{~mL}$ red gasoline sample passed slowly through the adsorbent (to ensure that the adsorbent can fully adsorb the red substance). After all the faded gasoline was discharged, the adsorbent became red or even brown (when the amount of adsorbed red substance was very large). The chromatographic column was washed with a cyclohexane and acetone mixture (with a cyclohexane to acetone volume ratio of 15:1) as eluant. The eluant was collected and then concentrated by vacuum distillation. Chromatographic separation of the concentrated eluant was repeated and a pure red substance was obtained.

\subsection{GC-MS and elemental analysis of red substance in the FCC gasoline}

With gas chromatography-mass spectrometry (GCMS) (5973N/6890, Agilent), the purified red substance was analyzed to determine the components of the compounds which caused the color change of gasoline. Conditions for GC-MS are as follows: Ionization mode, electron ionization (EI); Chromatographic column, DB5-MS $60 \mathrm{~m} \times 0.25 \mathrm{~mm} \times 0.25 \mu \mathrm{m}$; Initial column temperature, $50{ }^{\circ} \mathrm{C}$, being heated up to $280{ }^{\circ} \mathrm{C}$ at the rate of $4{ }^{\circ} \mathrm{C} / \mathrm{min}$, and kept at the temperature for 10 minutes; Vaporizing temperature, $300{ }^{\circ} \mathrm{C}$; Temperature of transfer line: $310{ }^{\circ} \mathrm{C}$; Column inlet pressure, $170 \mathrm{kPa}$; Split ratio, 50:1; Acquisition mode, Full scan.

With a Vario EL III elemental analyzer (Elementar Company, Germany), elemental analysis of the purified 
red substance was conducted, including carbon, hydrogen, nitrogen, and etc.

\subsection{Experiments on the red substance in FCC gasoline}

Understanding of the chemical properties of the red substance in the FCC gasoline is very important, thus reactions of the red substance with base, acid, oxidizer and others were carried out separately.

\section{Results and discussion}

\subsection{Analysis results of red substance in the FCC gasoline}

The GC-MS analysis results of the red substance concentrated by silica gel are shown in Fig. 1, Fig. 2 and Table 1. Fig. 3 is the mass spectrogram of standard N, N'-Disec-butyl-p-phenylenediamine (DBD). The GC-MS analysis results of the red substance concentrated by neutral alumina are shown in Fig. 4, Fig. 5 and Table 2.

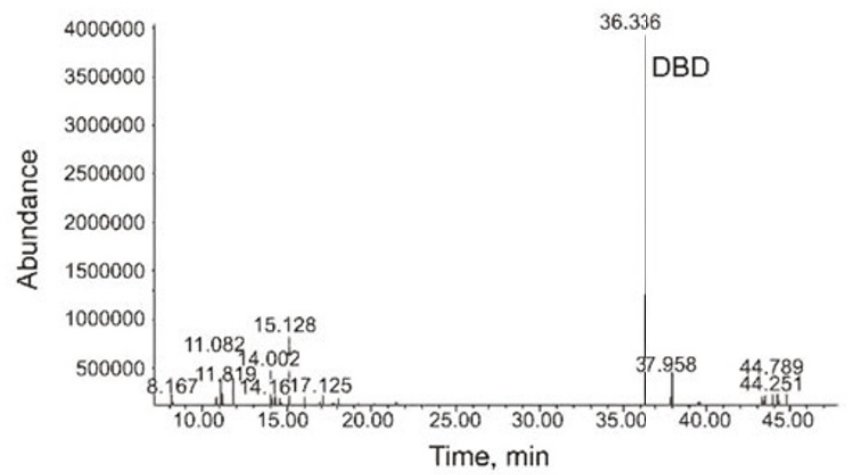

Fig. 1 GC-MS spectrum for the red substance adsorbed by silica gel

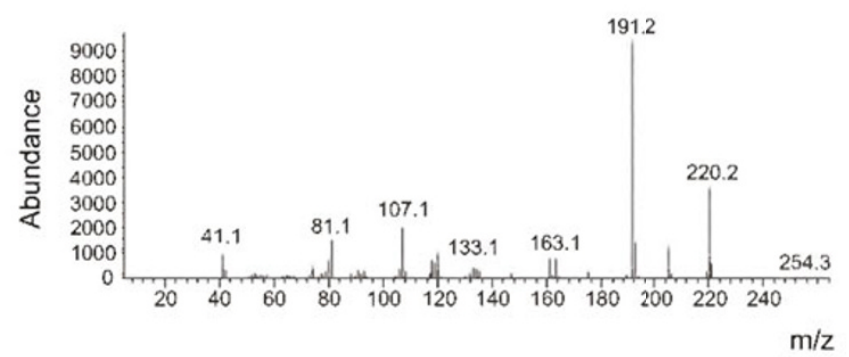

Fig. 2 Mass spectrogram of main components in the red substance adsorbed by silica gel

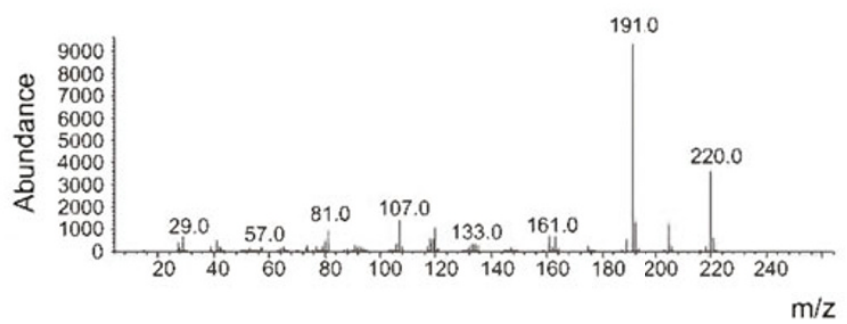

Fig. 3 Mass spectrogram of standard DBD
Table 1 GC-MS analysis result of the red substance adsorbed by silica gcl

\begin{tabular}{|c|c|c|c|}
\hline Number & Retention time, min & Substance & Relative content, $\%$ \\
\hline 1 & 8.167 & Methylbenzenc & 1.38 \\
\hline 2 & 11.082 & $\mathrm{C}_{2}$-benzene & 4.85 \\
\hline 3 & 11.819 & $\mathrm{C}_{2}$-benzene & 2.77 \\
\hline 4 & 14.002 & $\mathrm{C}_{3}$-benzene & 3.09 \\
\hline 5 & 14.287 & $\mathrm{C}_{3}$-benzene & 1.48 \\
\hline 6 & 15.128 & $\mathrm{C}_{3}$-benzene & 5.29 \\
\hline 7 & 16.050 & $\mathrm{C}_{3}$-benzene & 1.64 \\
\hline 8 & 17.125 & $\mathrm{C}_{4}$-benzene & 1.93 \\
\hline 9 & 36.336 & $\begin{array}{l}\text { N, N'-Di-sec-butyl-p- } \\
\text { phenylenediamine }\end{array}$ & 66.75 \\
\hline 10 & 37.958 & $\mathrm{C}_{10} \mathrm{H}_{22} \mathrm{O}_{4}$ esters & 2.82 \\
\hline 11 & 43.968 & $\mathrm{C}_{20} \mathrm{H}_{28} \mathrm{~N}_{2} \mathrm{O}$ indoles & 3.11 \\
\hline 12 & 44.251 & $\mathrm{C}_{18} \mathrm{H}_{20} \mathrm{~N}_{2} \mathrm{OS}$ thiophenes & 1.80 \\
\hline 13 & 44.789 & $\mathrm{C}_{18} \mathrm{H}_{18} \mathrm{~N}_{2} \mathrm{O}_{2} \mathrm{~S}$ thiophenes & 3.07 \\
\hline
\end{tabular}

Notes: $C_{2}$-benzene means that a benzene ring has different replacement with 1 or 2 position substituents replaced with total 2 carbon atoms. $\mathrm{C}_{3}-$ benzene and $\mathrm{C}_{4}$-benzene are the same as $\mathrm{C}_{2}$-benzene.

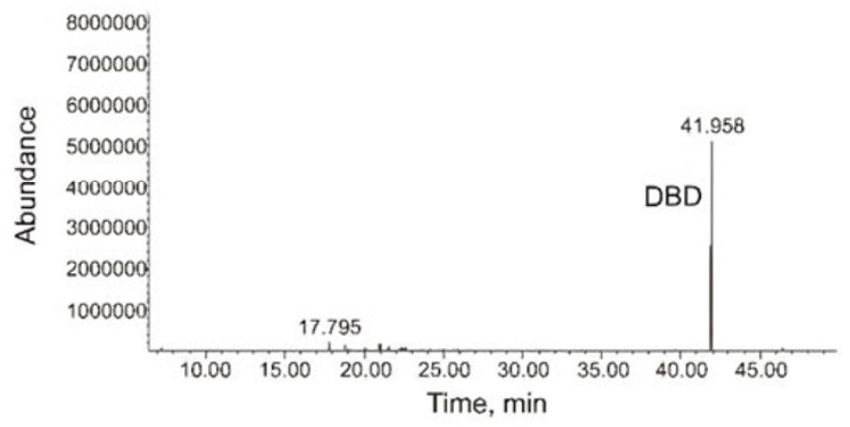

Fig. 4 GC-MS spectrum for the red substance adsorbed by neutral alumina

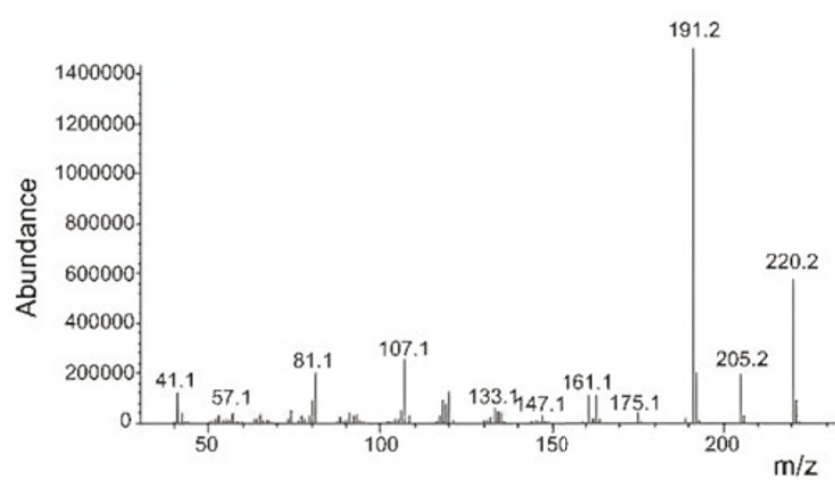

Fig. 5 Mass spectrogram of main components in the red substance adsorbed by neutral alumina 
Table 2 GC-MS analysis result of the red substance adsorbed by neutral alumina

\begin{tabular}{cccc}
\hline Number & Retention time, min & Substance & Relative content, \% \\
\hline 1 & 17.795 & $\mathrm{C}_{1}$-phenol & 2.92 \\
2 & 18.761 & $\mathrm{C}_{1}$-aniline & Trace \\
3 & 20.075 & $\mathrm{C}_{2}$ - phenol & Trace \\
4 & 24.982 & $\mathrm{C}_{3}$ - phenol & Trace \\
5 & 28.194 & $\mathrm{C}_{4}$ - phenol & Trace \\
6 & 41.958 & $\begin{array}{c}\text { N,N'-Di-sec-butyl-p- } \\
\text { phenylenediamine }\end{array}$ & 97.08 \\
\hline
\end{tabular}

Notes: $\mathrm{C}_{2}$-phenol means that the benzene ring of phenol has different replacement positions with 1 or 2 substituents replaced with total 2 carbon atoms. Others are the same as $\mathrm{C}_{2}$-phenol.

Silica gel and neutral alumina are different adsorbents and have different adsorption ability for the same red gasoline. From Fig. 1 and Table 1 it can be seen that the red substance concentrated by silica gel are mainly DBD, oxygen, nitrogen organic compounds and some aromatics. The results show that the red substance is a mixture of many components. By comparing Fig. 2 with Fig. 3, the main component of the red substance is DBD, which is also proved by Fig. 3 and Fig. 5. From Fig. 4 and Table 2 it can be seen that the red substance concentrated by neutral alumina are mainly DBD and $\mathrm{C}_{1}$ phenol. The results further show that the main components of the red substance are DBD and phenols. The analysis result of the red substance concentrated by silica gel is accordance with that concentrated by neutral alumina. Since trace of alkyl benzenes, esters, indoles and thiophenes does not show red, with the above analysis result, it can be concluded that the red color of gasoline is attributed to DBD and phenols, namely aromatic amines and phenols are responsible for the red color of gasoline. In common, the aromatic amines and phenols are the gasoline constitutes, however, why does DBD account for the most of the aromatic amines? Generally DBD as an antioxidant is added to gasoline for prolonging the induction period. It is deduced that when the content of aromatic amines and phenols is higher than the common value, they may be oxidized and interact with each other, leading to the red color of gasoline.

Elemental analysis of the red substance adsorbed by silica gel from the red gasoline is shown in Table 3 .

Table 3 Elemental analysis of the red substance

\begin{tabular}{cccccc}
\hline Element & $\mathrm{H}$ & $\mathrm{C}$ & $\mathrm{N}$ & $\mathrm{S}$ & $\mathrm{O}$ \\
\hline Content, \% & 10.31 & 76.20 & 7.14 & 1.10 & 5.25 (by difference) \\
\hline
\end{tabular}

Normally the nitrogen content in gasoline does not exceed $1000 \mathrm{ppmw}$ and the upper oxygen limit in gasoline is $2.7 \%$ (Chen, 1999). However the data in Table 3 shows that the contents of element $\mathrm{N}$ and $\mathrm{O}$ in the red substance are relatively high. It suggests that the main elements that lead to the color instability of gasoline are $\mathrm{N}$ and $\mathrm{O}$. Combined with the above GC-MS analysis result, the main components of the red substance in red gasoline are further determined as aromatic amines, phenols and their interaction compounds.

\subsection{Chemical properties of the red substance in FCC gasoline}

\subsubsection{Reaction between red gasoline and alkalis}

Red gasoline was added to a beaker, and then an alkali solution was added and the mixture stirred for 5 minutes. The observed results are presented in Table 4 .

Table 4 Phenomena of the reaction between red gasoline and alkalis

\begin{tabular}{cccc}
\hline Volume of red gasoline, $\mathrm{mL}$ & Alkali used & Proportion of alkali and gasoline & Observations \\
\hline 100 & $10 \mathrm{wt} \%$ caustic solution & $1: 10$ & Layer of oil remained red, caustic layer almost clear \\
100 & $5 \mathrm{wt} \%$ caustic solution from refinery & $1: 10$ & Same as above \\
100 & $2.2 \mathrm{wt} \%$ ammonia aqueous solution & $1: 20$ & Same as above \\
\hline
\end{tabular}

From Table 4, it can be seen that the red gasoline is not faded by alkalis, indicating that the red substance in the FCC gasoline does not react with alkalis. It could be deduced that the red substance is neutral or alkaline material.

\subsubsection{Reactions between red gasoline and acids}

Inorganic and organic acids, including hydrochloric, phosphoric, acetic, and propionic acid were chosen for experiment. Acid was separately added into red gasoline, and then stirred for 5 minutes. The results are shown in Table 5 .

Table 5 shows that the red gasoline can be faded with acids, indicating that the red substance in the FCC gasoline reacts with acids. It is definite that the red substance is alkaline material, and the alkaline substance in gasoline is organic alkalis, which are generally organic amines. Since hydrochloric acid and phosphoric acid are strong acids and are effective at washing nitrogen and oxygen compound impurities from gasoline, so the gasoline layer became colorless after the impurities reacted with these acids. The organic acids such as acetic and propionic acids are weak, and react slowly with the organic amines. So the color of the gasoline layer changed slowly when it was mixed with these acids. Further experiments were also conducted using tert-butyl hydroperoxide, sodium hypochlorite, and hydrogen peroxide as oxidizers, and found that the red oil could be faded, which demonstrated that the red substance in gasoline can be oxidized. 
Table 5 Phenomena during the reaction between red gasoline and acids

\begin{tabular}{|c|c|c|c|}
\hline Acid & $\begin{array}{c}\text { Volume of red } \\
\text { gasoline, } \mathrm{mL}\end{array}$ & $\begin{array}{l}\text { Volume of acid } \\
\qquad \mathrm{mL}\end{array}$ & Experimental phenomena \\
\hline Hydrochloric acid, $10 \mathrm{~mol} / \mathrm{L}$ & 25 & 1 & $\begin{array}{l}\text { The color of the gasoline layer deepened at the moment of adding acid, } \\
\text { then the red color disappeared rapidly, and the gasoline became clear }\end{array}$ \\
\hline Phosphoric acid & 25 & 1 & The gasoline layer became clear, and no change for a period of time \\
\hline Acetic acid & 25 & 1 & $\begin{array}{l}\text { The color of the gasoline layer deepened at the moment of adding acid, } \\
\text { and then became lighter gradually, and turned orange in } 5 \text { minutes }\end{array}$ \\
\hline Propionic acid & 25 & 1 & The color of the gasoline layer turned from red to yellow gradually \\
\hline
\end{tabular}

\subsection{Mechanism for color change of the FCC gasoline}

\subsubsection{Origin of red color of gasoline}

According to the report in literature (Wu et al, 2008b), phenols and aromatic amines are unstable components in FCC gasoline. They seriously affect the storage stability of gasoline, and even lead to darkening if their content is high. It is demonstrated above that the red substance in the FCC gasoline is composed of organic aromatic amines. In addition, the aromatic amines exist as complex compounds, not as free molecules, since the individual phenols and aromatic amines are colorless. Based on GC-MS and elemental analysis and in consideration of the red substance can react with acids, the origin of red color in the FCC gasoline was proposed as Scheme 1. Firstly, phenols and some aromatic amines were oxidized to quinones separately. Then, phenols and quinones act as electron donors and acceptors respectively. Organic amines and quinones could form weak charge transfer complexes (CTC), which have been reported to be red (Chen and Miao, 2003; Gao et al, 2003; Guo et al, 2003; Ma and Luo, 1995; Walters et al, 1948).

The red substance produced through oxidation and complexation in gasoline is the main factor for the red of gasoline. Gasoline will turn red if the concentration of complex is high enough. The higher the concentration of phenols and aromatic amines in gasoline, the more complexes of aromatic amines and quinones form. The formed charge transfer complex is not stable and may be decomposed into organic amines and quinones again when the temperature is high. Therefore, the red complex was decomposed when it

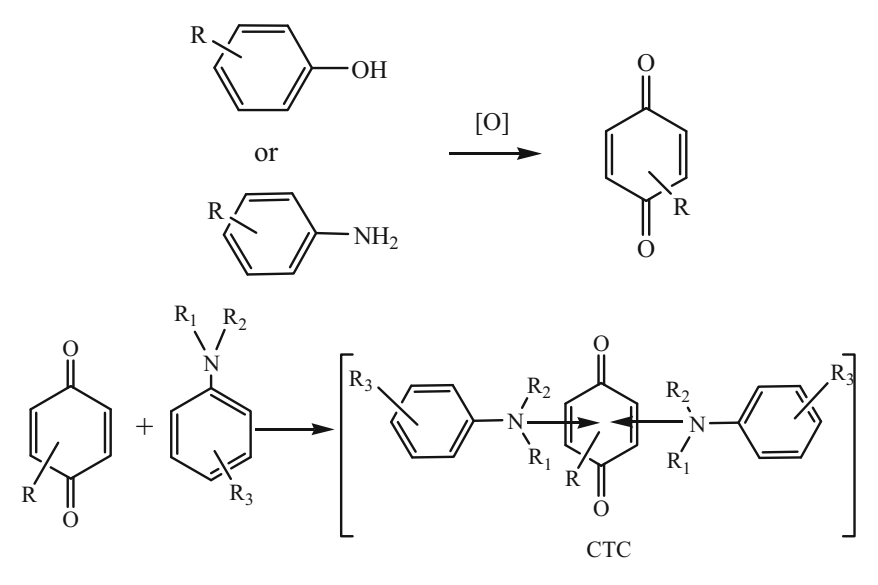

Scheme 1

was analyzed with GC-MS, and the DBD was found in the compounds.

\subsubsection{Reason why red color fading when FCC gasoline processed with acids}

As observed above, the red faded quickly when acids were added to the red gasoline. This result should be caused by the destruction of the charge transfer complex due to the presence of acids. In addition, organic acids and inorganic acids all can fade red gasoline, indicating that the reaction is related to the protons of acids, not to anions of acids. Amines in the complex are alkaline, and can react with acids to form amine salts, which lead to the breakdown of the complex as shown in Scheme 2 (Ci et al, 1989).

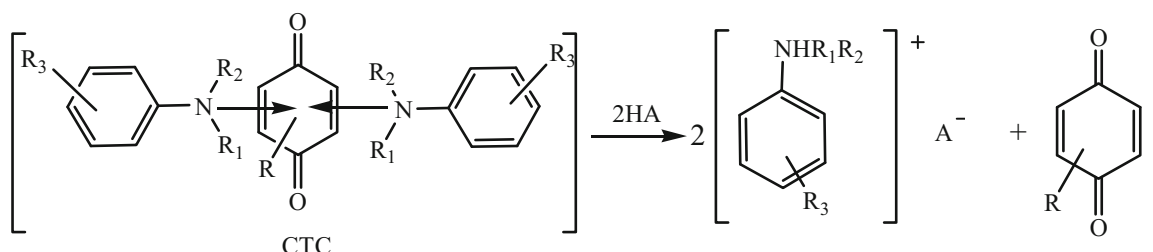

Scheme 2

\section{Conclusions}

Aromatic amines were found to be the main components of the red substance separated from FCC gasoline. The fading of red FCC gasoline with acids further demonstrated that the aromatic amines were in the form of complexes in the gasoline. Phenols and some of the aromatic amines were first oxidized to quinones, and then the red complex was formed by the reaction of unoxidized aromatic amines and quinones. The complex was decomposed by acids and simultaneously the red color of the gasoline disappeared.

\section{Acknowledgements}

This research was supported by the Fundamental Research 
Funds for the Central Universities (No. 10CX04024A).

\section{References}

Andziulis A. Long-term oxidation stability of gasoline on account of MIR monitoring. Transport. 2006. 21(3): 218-222

Batts B D and Fathoni A Z. A literature review on fuel stability studies with particular emphasis on diesel oil. Energy \& Fuels. 1991. 5(1): $2-21$

Chen $\mathrm{G} R$ and Miao Y. Charge-transferring reaction between sulfaguanidine and tetrachlorobenzoquinone. Physical Testing and Chemical Analysis Part B Chemical Analysis. 2003. 39(9): 535-536 (in Chinese)

Chen X K. World fuel specifications \& Chinese standard for new formula gasoline. Petroleum Products Application Research. 1999. 17(3): 11-13 (in Chinese)

Ci X H, Silva R S, Nicodem D, et al. Electron and hydrogen atom transfer mechanisms for the photoreduction of o-quinones. Visible light induced photoreactions of P-lapachone with amines, alcohols, and amino alcohols. Journal of the American Chemical Society. 1989. 111(4): 1337-1343

Gao Z R, Liu D S and Liao K J. Study on improving invariability of gasoline by ILS catalyst. Liaoning Chemical Industry. 2003. 32(11): 482-485 (in Chinese)

Guo H L, Yan B Z and Guo X Y. Photo-CIDNP study of electron transfer reaction of triethylamine with 2-Chloro-5-methoxy-p-benzoquinone. Chinese Journal of Organic Chemistry. 2003. 23(3): 288-290 (in
Chinese)

Ma C X and Luo H J. Compendious analysis for colored mechanism of organic substances. Journal of Daqing Petroleum Institute. 1995. 19(1): 47-50 (in Chinese)

Pereira R C C and Pasa V M D. Effect of mono-olefins and diolefins on the stability of automotive gasoline. Fuel. 2006. 85(12): 1860-1865

Thompson R B, Chenicek J A and Druge L W. Stability of fuel oils in storage: Effect of some nitrogen compounds. Industrial \& Engineering Chemistry. 1951. 43(4): 935-939

Thompson R B, Druge L W and Chenicek J A. Stability of fuel oils in storage: Effect of sulfur compounds. Industrial \& Engineering Chemistry. 1949. 41(12): 2715-2721

Walters E L, Yabroff D L and Minor H B. Correlation of predicted and observed storage stability of cracked gasoline. Industrial \& Engineering Chemistry. 1948. 40(3): 423-428

Wu S K, Liang C L, Huang K M, et al. Effects of components of FCC gasoline on its storage stability. Acta Petrolei Sinica (Petroleum Processing Section). 2008a. 24(4): 478-483 (in Chinese)

Wu S K, Xu B F and Liu B S. Impact factor analysis of color stability of FCC naphtha and countermeasures. Petroleum Refinery Engineering. 2008b. 38(8): 59-62 (in Chinese)

Zanier A. Thermal-oxidative stability of motor gasolines by pressure d.s.c. Fuel. 1998. 77(8): 865-870

Zuo L S. The studies on color stability of catalytically cracked diesel oil. MS Dissertation. Hu nan: Hunan University. 2005 (in Chinese)

(Edited by Zhu Xiuqin) 